\title{
Tuning Anisotropy Barriers in a Family of Tetrairon(III) Single-Molecule Magnets with a $S=5$ Ground State
}

Stefania Accorsi,$^{\dagger}$ Anne-Laure Barra, ${ }^{\ddagger}$ Andrea Caneschi, ${ }^{\S}$ Guillaume Chastanet, ${ }^{\S}$ Andrea Cornia, ${ }^{*}{ }^{\dagger}$ Antonio C. Fabretti,,$^{\dagger}$ Dante Gatteschi, ${ }^{\S}$ Cecilia Mortalo ${ }^{\dagger}$ Emiliano Olivieri,${ }^{\S}$ Francesca Parenti, ${ }^{\dagger}$ Roberta Sessoli, ${ }^{*} \S$ Lorenzo Sorace, ${ }^{\S}$ Wolfgang Wernsdorfer, ${ }^{\text {II }}$ and Laura Zobbi ${ }^{\dagger}$

Contribution from the INSTM \& Department of Chemistry, University of Modena and Reggio Emilia, via G. Campi 183, 41100 Modena, Italy, Laboratoire des Champs Magnetiques Intenses-CNRS, BP166, 25 Avenue des Martyrs, 38042 Grenoble Cedex 9, France, INSTM \& Department of Chemistry, University of Florence, via della Lastruccia 3, 50019 Florence, Italy, and Laboratoire Louis Néel-CNRS, BP166, 25 Avenue des Martyrs, 38042 Grenoble Cedex 9, France

E-mail: $\underline{\text { acornia@ unimore.it; roberta.sessoli@unifi.it }}$

\section{Supporting Information}




\section{Details of X-ray Work}

$\left[\mathrm{Fe}_{4}\left(\mathbf{L}^{\mathbf{1}}\right)_{\mathbf{2}}(\mathbf{d p m})_{6}\right]$ (1). The selected sample was mounted on a glass fiber with epoxy resin for diffraction measurements. The trigonal crystal system, suggested by the cell metrics, was confirmed by the $\overline{3} m$ Laue symmetry of the diffraction pattern. Systematic absences $h k l:-h+k+l \neq 3 n$ and $h-h l: l \neq 2 n$ ( $n$ integer) were consistent with space groups $R 3 c$ (No. 161, non centrosymmetric) and $R \overline{3} c$ (No. 167, centrosymmetric). Intensity statistics strongly suggested a centrosymmetric space group $\left(\left|E^{2}-1\right|=0.915\right.$ vs. $0.968 / 0.736$ for a centrosymmetric/non-centrosymmetric space group), as confirmed by the successful structure solution and refinement in space group $R \overline{3} c$. One ${ }^{t} \mathrm{Bu}$ group was disordered over two positions with Site Occupancy Factors (SOFs) 0.849(5) and 0.151(5). All nonhydrogen atoms, except for those of the disordered ${ }^{t} \mathrm{Bu}$ in the lower-occupancy position, were refined anisotropically. Hydrogen atoms were added in idealized positions, with torsion angle refinement for Me groups, and treated isotropically with $U_{\mathrm{eq}}=K \cdot U_{\mathrm{eq}}(\mathrm{C})$ were $K=1.5$ for $\mathrm{CH}_{3}$ and $K=1.2$ for $\mathrm{CH}_{2}$ and $\mathrm{CH}$ groups.

$\left[\mathrm{Fe}_{4}\left(\mathbf{L}^{3}\right)_{2}(\mathbf{d p m})_{6}\right] \cdot \mathbf{E t}_{2} \mathbf{O}\left(\mathbf{3} \cdot \mathrm{Et}_{2} \mathrm{O}\right)$. The sample used for the data collection, cut from a larger individual, was removed from the mother liquid, mounted on a glass fiber with a trace of vacuum grease and rapidly transferred into the cold nitrogen stream of the diffractometer. Cell constants were determined from the setting angles of 37 reflections at $10.27 \leq \theta \leq 14.24^{\circ}$ and the monoclinic crystal system was confirmed by the symmetry of the diffraction pattern (Laue group $2 / m$ ). Data collection was carried out with $\theta-2 \theta$ scans. No decay of standard intensity was observed. Systematic absences $h k l: h+k \neq 2 n$ and $h 0 l: h, l \neq$ $2 n$ ( $n$ integer) suggested space groups $C c$ (No. 9, non centrosymmetric) or $C 2 / c$ (No. 15, centrosymmetric). $E$-statistics strongly suggested a centrosymmetric space group $\left(\left|E^{2}-1\right|=0.974 v s\right.$. 0.968/0.736 for a centrosymmetric/non-centrosymmetric space group), as confirmed by successful structure solution and refinement in space group $C 2 / c$. A ${ }^{t} \mathrm{Bu}$ group was disordered over two positions with SOFs 0.561(13) and 0.439(13). The unit cell contains one $\mathrm{Et}_{2} \mathrm{O}$ molecule per cluster, which was modelled with full occupancy. The density calculated from the room-temperature unit-cell volume [10111(5) $\left.\AA^{3}\right]$ is $1.153 \mathrm{~g} / \mathrm{cm}^{3}$, in nice agreement with the experimental value obtained by flotation in 
aqueous $\mathrm{NaBr}, 1.15(1) \mathrm{g} / \mathrm{cm}^{3}$. All nonhydrogen atoms were treated anisotropically, whereas hydrogen atoms were handled as described for $\mathbf{1 .}$

$\left[\mathrm{Fe}_{4}(\mathbf{O E t})_{3}\left(\mathbf{L}^{\mathbf{4}}\right)(\mathbf{d p m})_{6}\right](\mathbf{4})$. The selected sample was mounted on a glass fiber with a trace of vacuum grease and transferred to the cold nitrogen stream of the diffractometer. The cell metrics and the diffraction pattern indicated a trigonal crystal system, with $R($ int $)=0.030$ and 0.032 in Laue classes $\overline{3}$ and $\overline{3} m 1$, respectively (see Figure S1). Systematic absences were indicative of a $R$-centered lattice (hexagonal axes), i.e. $h k l:-h+k+l \neq 3 n$, pointing to space groups $R 3$ (No. 146) or $R \overline{3}$ (No. 148) in Laue class $\overline{3}$, and to space groups $R 32$ (No. 155), $R 3 m$ (No. 160) or $R \overline{3} m$ (No. 166) in Laue class $\overline{3} m 1$. From the density expected for this class of compounds $\left(\sim 1.2 \mathrm{~g} / \mathrm{cm}^{3}\right)$, it was calculated that $Z=6$. Space groups $R 3 m$ and $R \overline{3} m$ were then discarded because, in these space groups, sites with multiplicity 6 or lower have $3 m$ or $\overline{3} m$ symmetry, which is incompatible with the expected molecular structure. $E$ statistics did not clearly indicate the centricity, so that structure solution was attempted in the three remaining space groups. In fact, the structure was solved and refined successfully in space group $R \overline{3}$. The cluster was found to develop around a threefold axis and location of all nonhydrogen atoms was straightforward. However, $R 1$ was as high as 0.2978 for isotropic refinement, but dropped to 0.1252 when merohedral twinning by two-fold rotation around the $a$-axis was assumed (TWIN $100-10-1000$ -12 ). The twinning fraction was very close to $50 \%$, thus explaining the $\overline{3} m 1$ symmetry of the diffraction pattern. After inclusion of hydrogen atoms in idealized positions and proper weighing, the model converged to $R 1=0.0822$. Electron density residuals $\left(0.8-0.5 \mathrm{e} / \AA^{3}\right)$ in Fourier difference maps revealed a fraction of dpm ligands adopting a different coordination mode, as previously found in $\mathbf{5}^{[7]}$ (see Figure S2). Disorder was best modelled with occupancy factors 0.82 and 0.18 , treating the minor component isotropically (with two overall thermal parameters for the carbon atoms of Me groups and for the remaining carbon atoms) and constraining its geometry to that of the maior component. The largest electron density residues $\left(2.8 \mathrm{e} / \AA^{3}\right)$ was located on the threefold axis at $1.0 \AA$ from $\mathrm{Fe} 1$, indicating the possible occurrence of additional disorder effects. 


\section{Details of ${ }^{1} \mathbf{H}$ and ${ }^{2} \mathbf{H}$ NMR Experiments.}

${ }^{1} \mathrm{H}$ NMR spectra have been collected on $15-\mathrm{mM}$ and $9.3-\mathrm{mM}$ solutions of 5 in toluene- $d_{8}$ and $\mathrm{CDCl}_{3}$, respectively, prepared by dissolving 11-mg and 7-mg samples of 5 in $0.5 \mathrm{~mL}$ of solvent.

${ }^{1} \mathrm{H}-\mathrm{NMR}$ spectra of freshly-prepared solutions show a very broad peak at 10.5-10.3 ppm (full-widthat-half-maximum $=3-4 \mathrm{ppm}$ ) and a much weaker signal at $-16 \mathrm{ppm}$. These resonances were assigned to the paramagnetically-shifted ${ }^{t} \mathrm{Bu}$ and methine protons of dipivaloylmethanide ligands, respectively. The spectra are time-dependent in that a shoulder just visible on the low-field side of the main peak in freshly-prepared solutions progressively gains intensity as an additional band at ca. 13 ppm. Correspondingly, the high-field peak of methine dpm protons splits into two signals at $c a$. -16 and -24 ppm. The peaks exhibiting the greatest paramagnetic shift (13 and $-24 \mathrm{ppm})$ are similar to those reported for $\mathrm{Fe}(\mathrm{dpm})_{3}$ in $\mathrm{CDCl}_{3},{ }^{16}$ pointing to progressive decomposition of the cluster to give magnetically uncoupled, monomeric species.

For the collection of deuteron spectra, crystalline 5-D $(38.7 \mathrm{mg}, 0.0239 \mathrm{mmol})$, carefully dried overnight over $\mathrm{P}_{2} \mathrm{O}_{5}$, was dissolved in anhydrous diethylether (Fluka, puriss. $\geq 99.8 \%$, dried over molecular sieve, $\mathrm{H}_{2} \mathrm{O} \leq 0.005 \%, 15 \mathrm{~mL}$ ), which required about $1 \mathrm{~h}$ of vigorous stirring. A $0.5-\mathrm{mL}$ aliquot of the resulting solution was immediately transferred to a 5-mm NMR tube and used to follow the solution behavior of the cluster. To two 5 -mL portions of the remaining solution, solid $\mathrm{H}_{3} \mathrm{~L}^{1}(2.58$ $\mathrm{mg}, 0.0215 \mathrm{mmol})$ and $\mathrm{H}_{3} \mathrm{~L}^{3}(3.96 \mathrm{mg}, 0.0217 \mathrm{mmol})$ were added with stirring to reach a $2.7: 1$ ligandto-cluster ratio. After complete dissolution of the ligands (30'), ${ }^{2} \mathrm{H}$ spectra were recorded on a $0.5-\mathrm{mL}$ sample of each solution. ${ }^{2} \mathrm{H}$ spectra were also recorded on solutions of 6-D $(14 \mathrm{mg})$ and 7-D $(10 \mathrm{mg})$ in $5 \mathrm{~mL}$ of dry diethylether.

Magnetic susceptibilities in solution were measured by Evans method. A 3\% (w/w) solution of $\mathrm{CDCl}_{3}$ in anhydrous $\mathrm{Et}_{2} \mathrm{O}$ (density $=0.714 \mathrm{~g} / \mathrm{mL}$ at $302 \mathrm{~K}$ ) was used to prepare solutions of 3-D.Et ${ }_{2} \mathrm{O}, \mathbf{5}-\mathbf{D}, \mathbf{6}-\mathbf{D}$ and 7-D with concentrations $5.94 \cdot 10^{-3}, 2.64 \cdot 10^{-3}, 3.67 \cdot 10^{-3}$ and $4.62 \cdot 10^{-3} \mathrm{~g} / \mathrm{mL}$, respectively. The same 3$\%$ solution was also used as an external standard. For all samples, the deuteron signals of $\mathrm{CDCl}_{3}$ in the two environments were separated by $\Delta \mathrm{v} / \mathrm{v}_{0}>0.25 \mathrm{ppm}$, while the FWHM of the signals was $<0.015$ 
ppm, thus enabling a precise evaluation of peak separation. The specific susceptibility of the paramagnetic solute was calculated by the formula:

$$
\chi_{\mathrm{g}}=\frac{3}{4 \pi m} \frac{\Delta \mathrm{v}}{\mathrm{v}_{0}}+\chi_{\mathrm{g}}^{\mathrm{solv}}
$$

which is valid for a superconducting solenoid configuration (magnetic field parallel to the long axis of cylindrical samples). ${ }^{12}$ In the above expression, $\chi_{\mathrm{g}}{ }^{\text {solv }}$ is the specific susceptibility of the solvent mixture (assumed to be equal to that of pure $\mathrm{Et}_{2} \mathrm{O}$, i.e. $-0.7434 \cdot 10^{-6} \mathrm{emu} / \mathrm{g}$ ) and $m$ is the solute concentration (in $\mathrm{g} / \mathrm{mL}$ ). The specific susceptibility was converted to molar susceptibility based on the following molecular masses (in u.m.a.): 1864.12 (3-D·Et ${ }_{2} \mathrm{O}$ ), 1617.85 (5-D), 979.28 (6-D) and 658.91 (7-D). Diamagnetic corrections (in $10^{-6} \mathrm{emu} / \mathrm{mol}$ ), estimated using Pascal's constants, amounted to -1021 (3D. $\left.\mathrm{Et}_{2} \mathrm{O}\right),-871($ 5-D), $-535($ 6-D) and -367 (7-D). The results are collected in Table S1 together with solid state values.

Details of DC Magnetic Measurements. Data reduction was based on molecular masses (in u.m.a.) of $1715.05(2), 1755.47\left(3 \cdot \mathrm{Et}_{2} \mathrm{O}\right)$ and $1617.35(4)$, and on sample weights (in $\mathrm{mg}$ ) of $20.45(2), 19.98$ $\left(3 \cdot \mathrm{Et}_{2} \mathrm{O}\right.$ ) and 6.25 (4). Diamagnetic corrections (in $10^{-6} \mathrm{emu} / \mathrm{mol}$ ), estimated using Pascal's constants, amounted to $-942(\mathbf{2}),-1021\left(3 \cdot \mathrm{Et}_{2} \mathrm{O}\right)$ and $-954(\mathbf{4})$.

For compound 4, intermolecular interactions have been introduced in the mean-field approximation. In the treatment of $\chi_{\mathrm{m}} T$-vs- $T$ data, the magnetic susceptibility given by eq. 2 has been corrected through the equation:

$$
\chi_{\mathrm{m}}^{\prime}=\chi_{\mathrm{m}}\left(1-\frac{z J^{\prime}}{N_{\mathrm{A}} g^{2} \mu_{\mathrm{B}}^{2}} \chi_{\mathrm{m}}\right)^{-1}
$$

where $J$ is the exchange constant with nearest-neighbors, whose number is $z\left(J \mathbf{S}_{i} \mathbf{S}_{j}\right.$ convention). ${ }^{19}$ In the treatment of magnetization-vs- $H$ data, the mean-field corrected magnetization was calculated as delineated in Reference 19c, solving numerically the self-consistent equation for $M_{\mathrm{m}}$. 


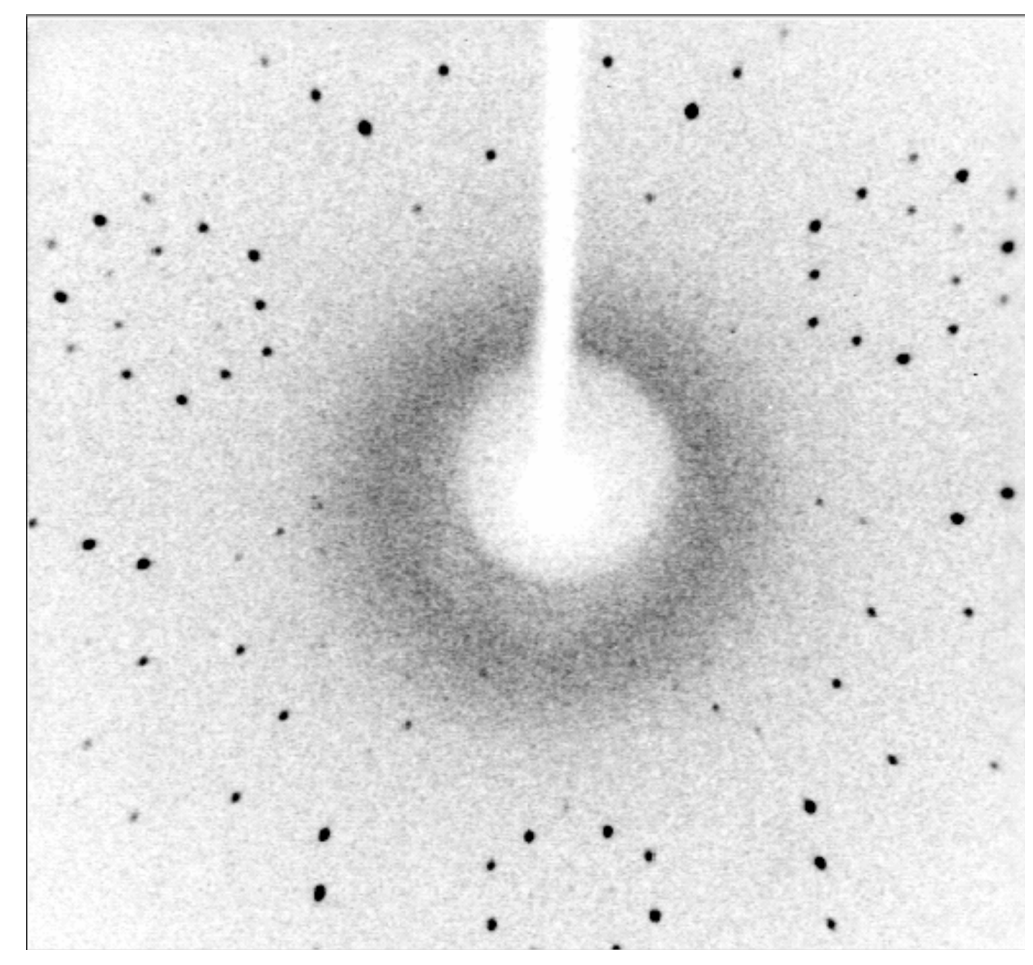

Figure S1. X-ray diffraction pattern of $\mathbf{4}$ recorded with the incident beam along the trigonal axis and showing apparent $3 m$ symmetry, consistent with Laue group $\overline{3} m 1$.

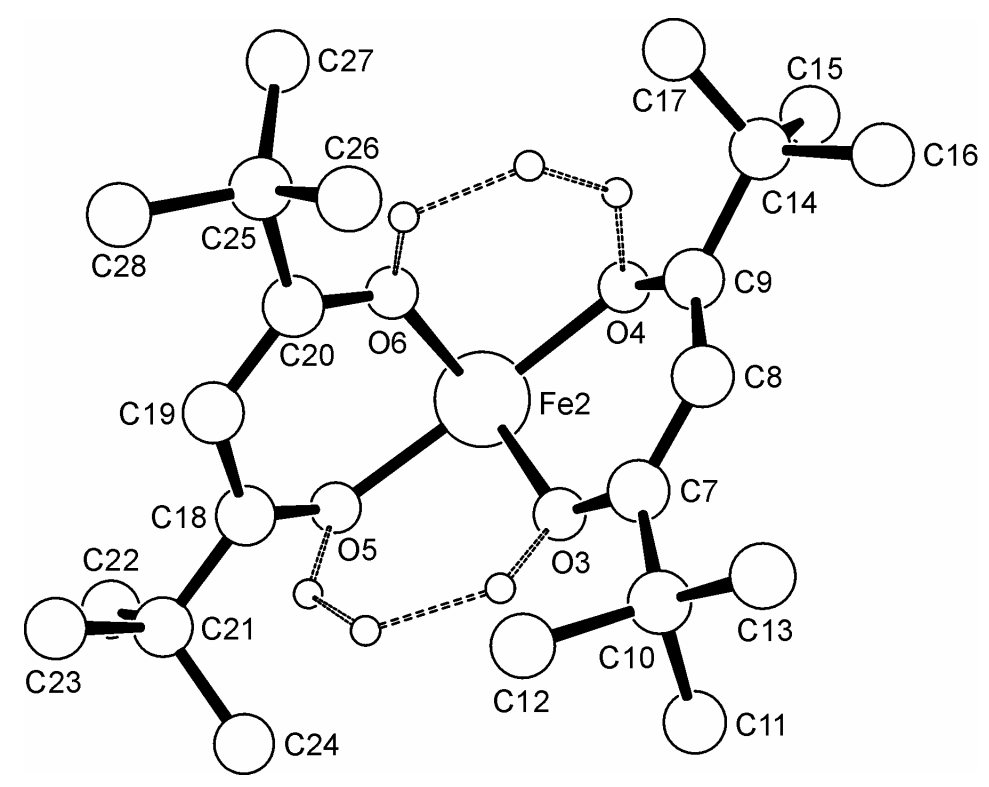

Figure S2. Electron density residuals (small spheres) found in the X-ray structure analysis of 4, showing the presence of disorder effects in the coordination sphere of Fe2. 


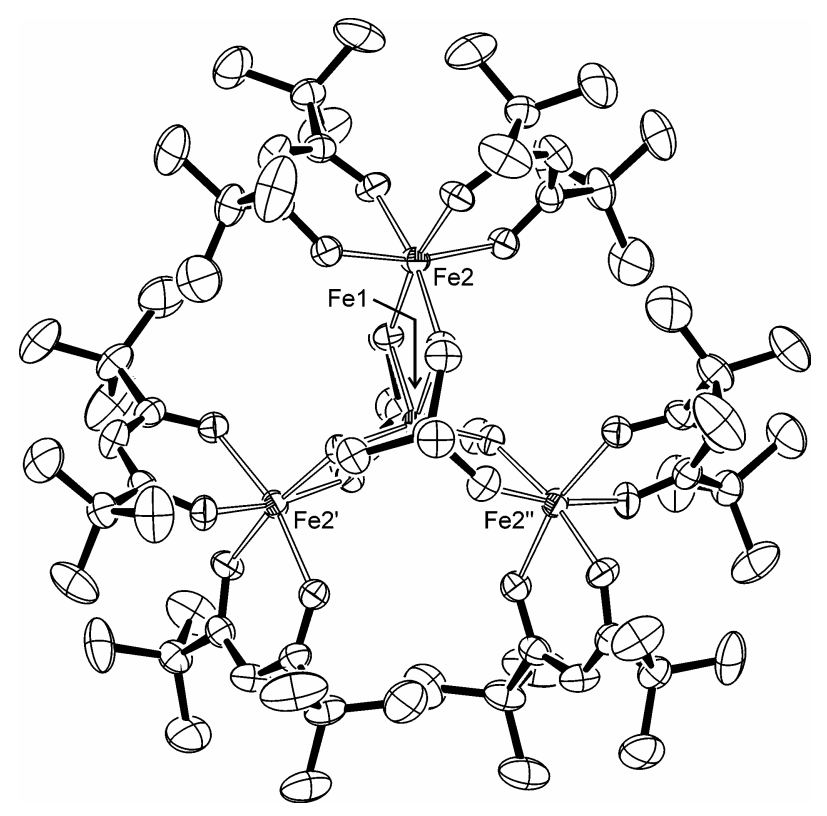

Figure S3. Molecular structure of $\mathbf{1 .}$

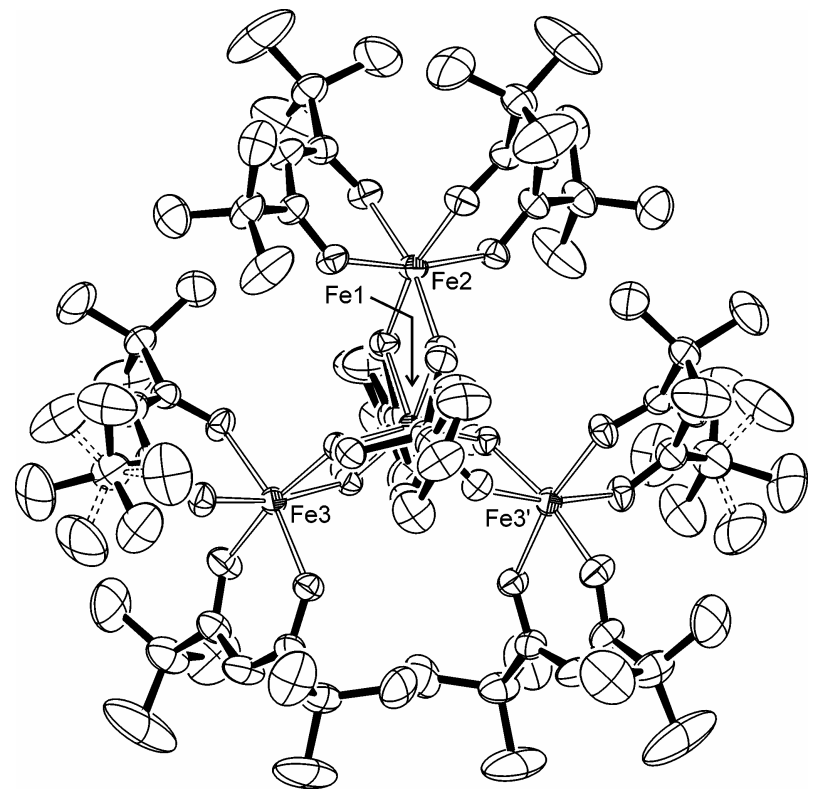

Figure S4. Molecular structure of 3. 


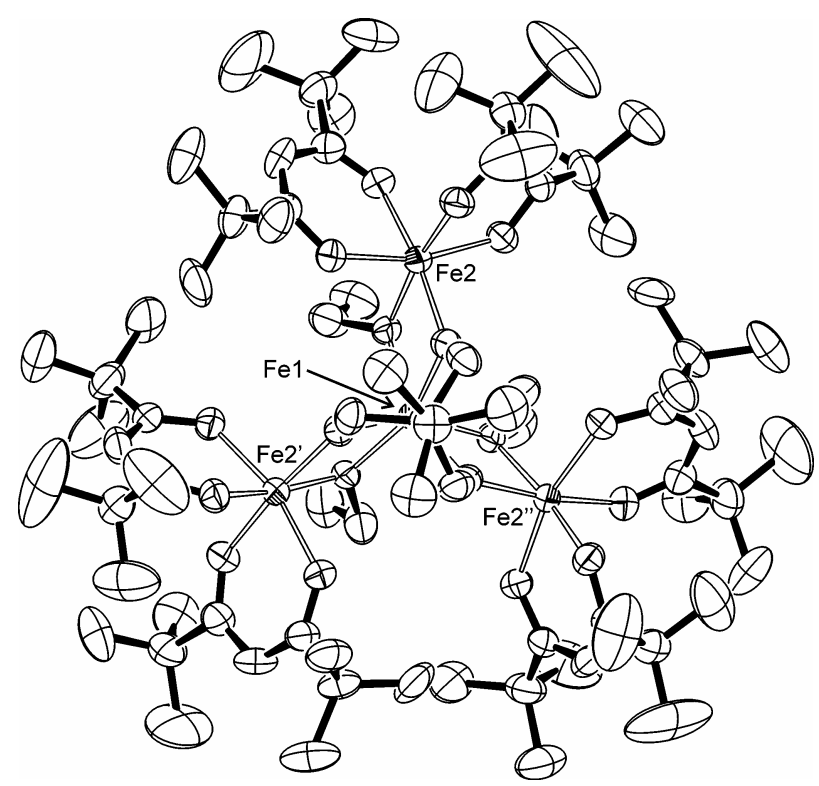

Figure S5. Molecular structure of 4 (isomer $a a a$ ).
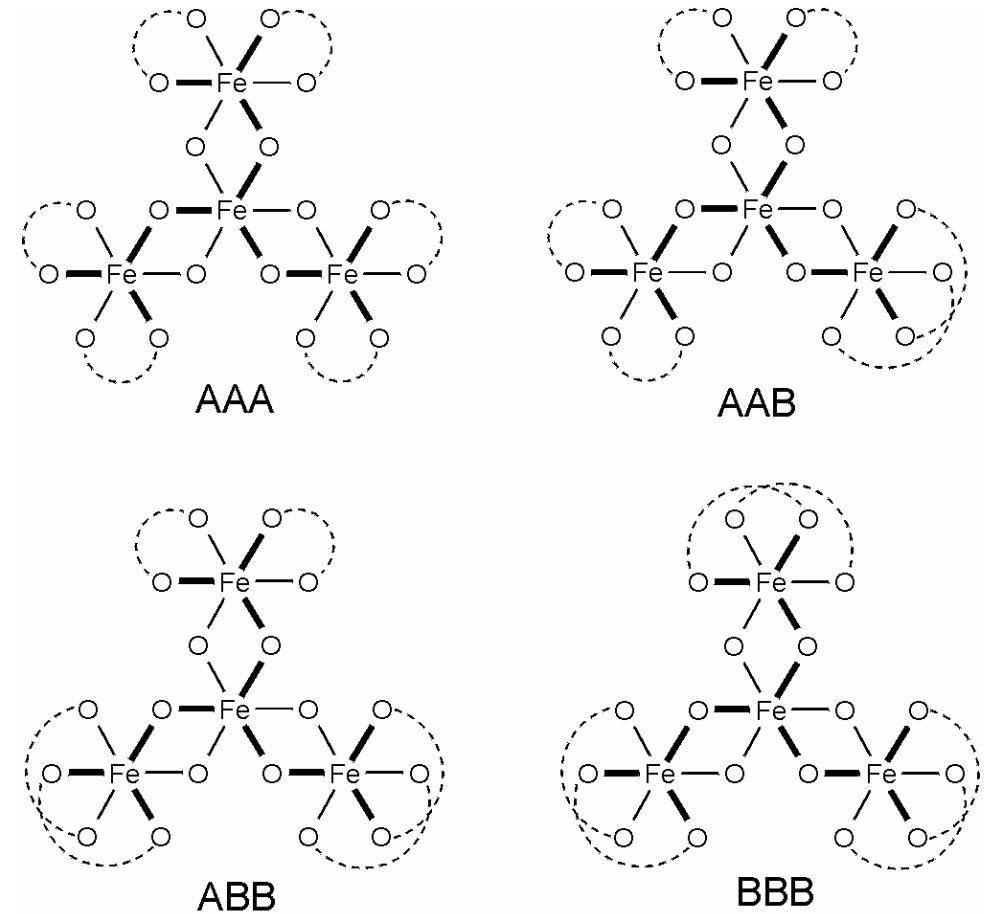

Figure S6. Sketched structures of geometric isomers $a a a, a a b, a b b$ and $b b b$ of 4 . 


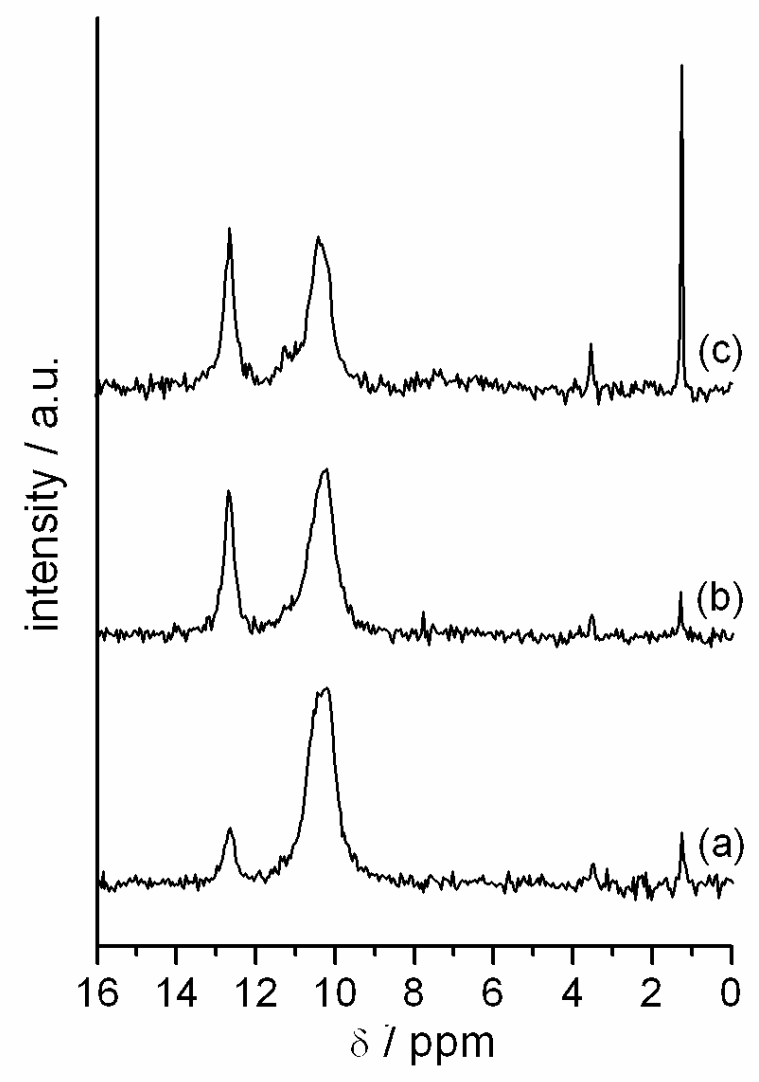

Figure S7. ${ }^{2} \mathrm{H}$ NMR spectra of 5-D in $\mathrm{Et}_{2} \mathrm{O}$ recorded at 40' (a), 330' (b) and 2 weeks (c) after complete dissolution and addition of solid $\mathrm{H}_{3} \mathrm{~L}^{1}(\mathrm{R}=\mathrm{Me})$.
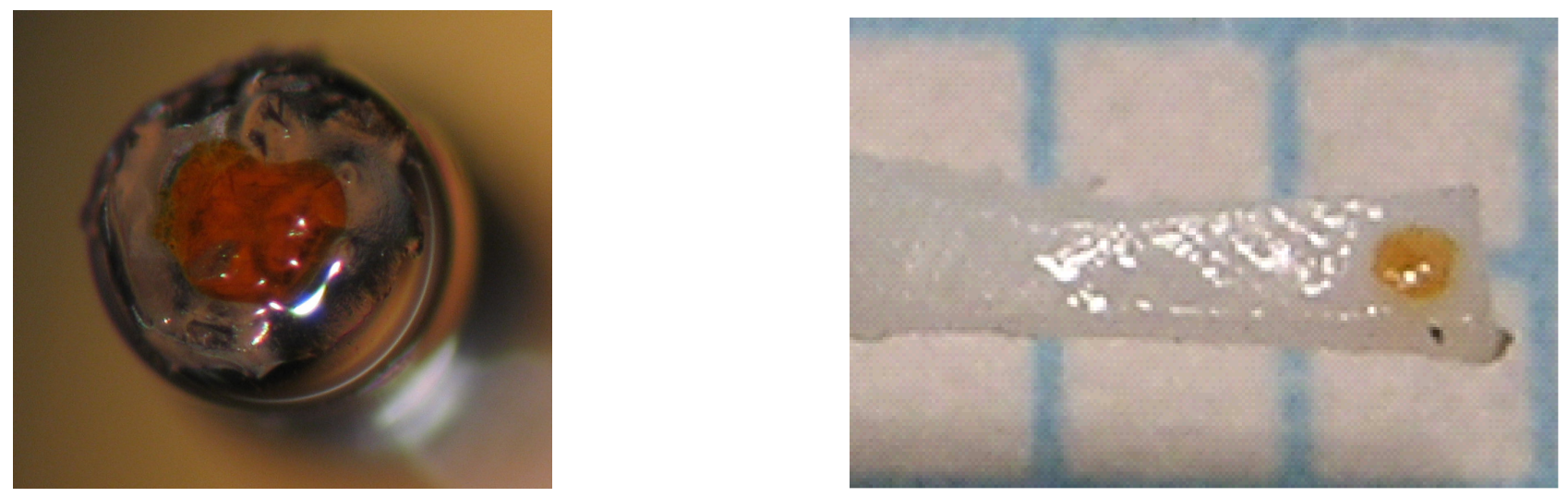

Figure S8. Single crystals of $\mathbf{4}$ mounted for W-Band EPR measurements in the $a b$ plane (left) and in an arbitrary plane containing $c$ axis (right). 


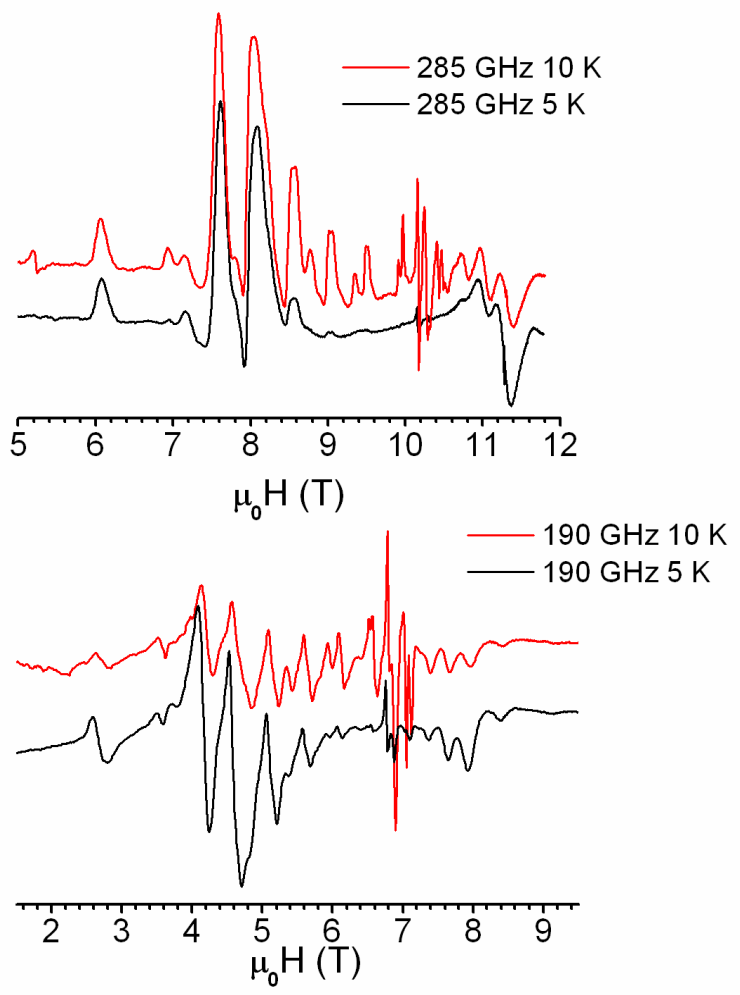

Figure S9. Low-temperature powder HF-EPR spectra of 4 at 190 and $285 \mathrm{GHz}$.

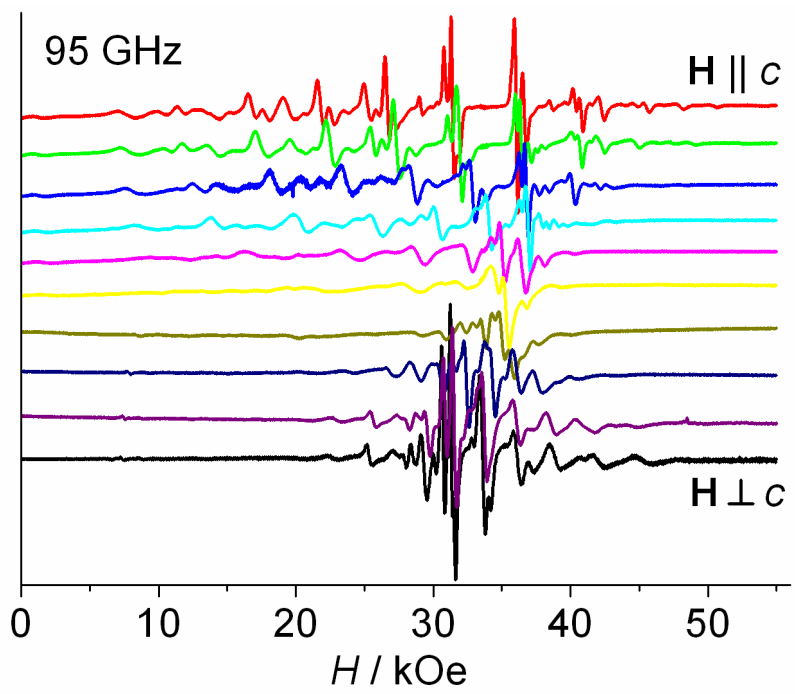

Figure S10. Angular dependence of W-Band EPR spectrum of a single crystal of 4 measured at $20 \mathrm{~K}$ from the easy axis (top) to the hard plane (bottom) in steps of $10^{\circ}$. 


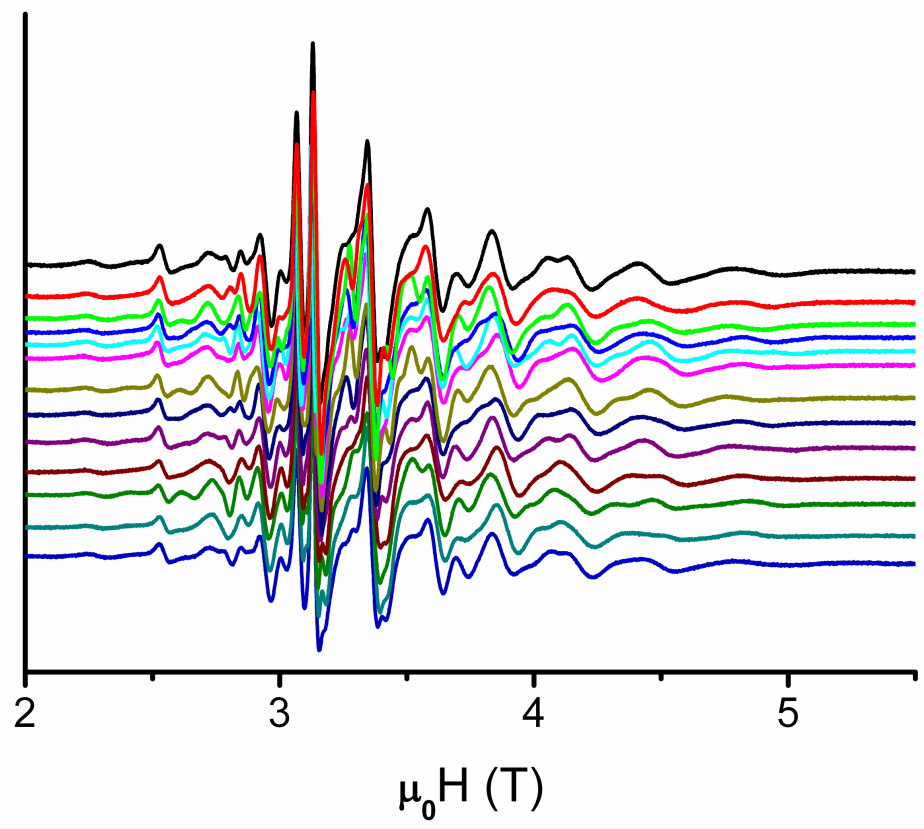

Figure S11. Single-crystal W-Band EPR spectra of 4 at $20 \mathrm{~K}$ with the magnetic field directed in the $a b$ plane. The spectra were recorded in steps of $15^{\circ}$ starting from an arbitrary direction and cover a range of $180^{\circ}$

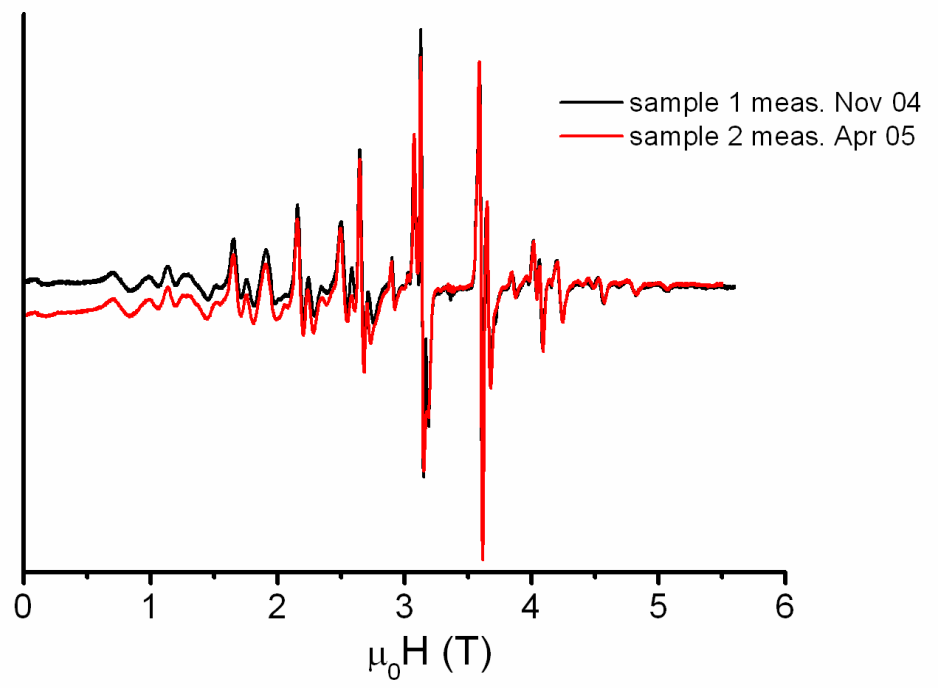

Figure S12. Single-crystal W-Band EPR spectra of 4 at $20 \mathrm{~K}$ recorded on two different samples. 


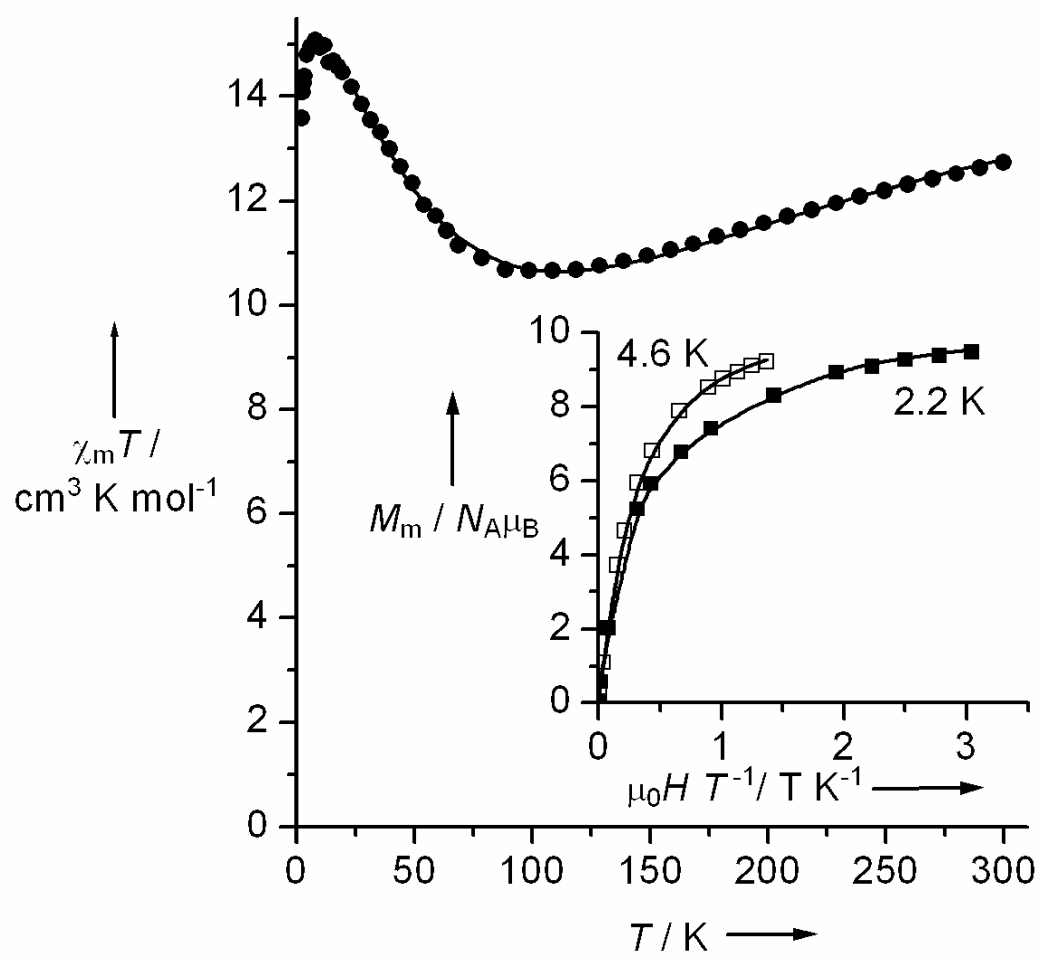

Figure S13. Magnetic behaviour of compound 3. $\mathrm{Et}_{2} \mathrm{O}$. The solid curves have been calculated using the best-fit parameters reported in the text.

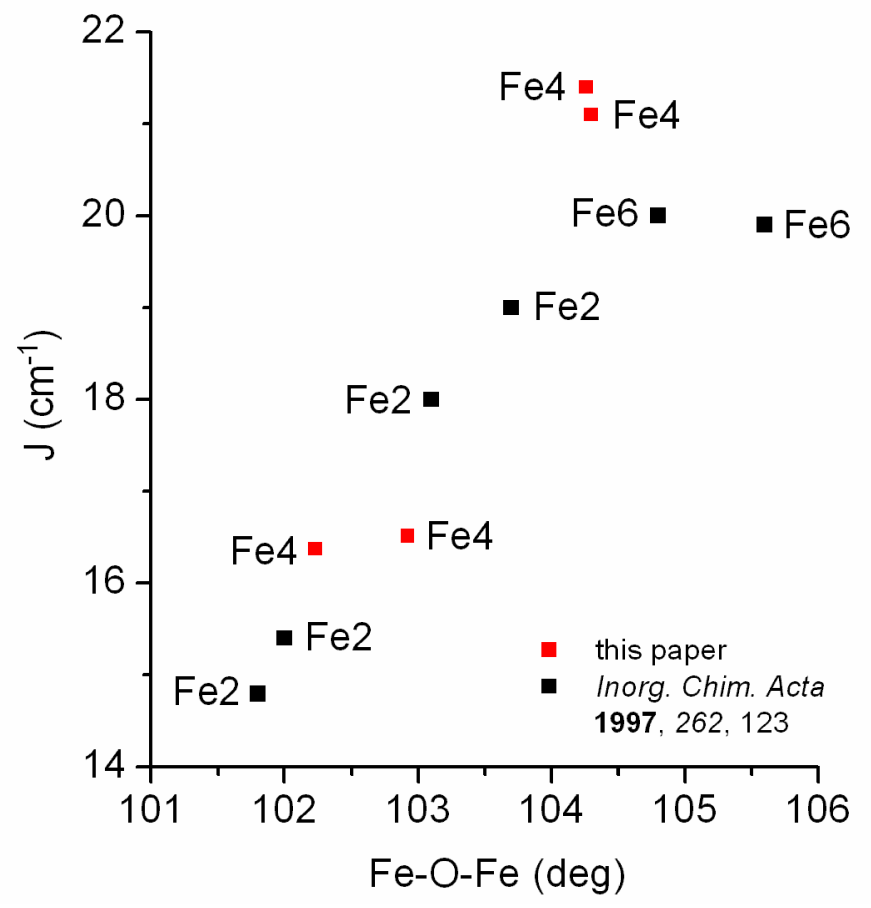

Figure S14. Plot of the experimental $J$ constant versus average Fe-O-Fe angle in bis(alkoxo)-bridged $\mathrm{Fe}_{2}$ units. 


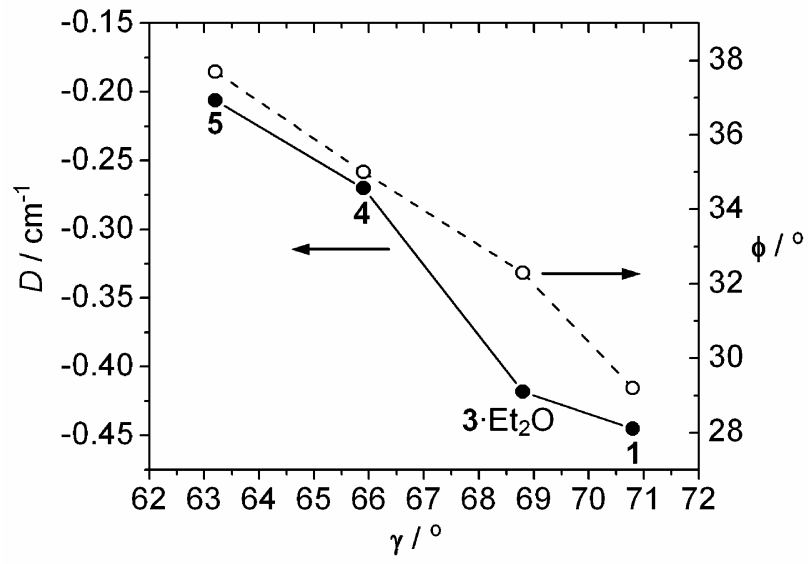

Figure S15. Correlation bewteen the helical pitch $(\gamma)$, the extent of distortion by trigonal rotation $(\phi)$ and the ZFS parameter $(D)$ in $\mathbf{1}, \mathbf{3} \cdot \mathrm{Et}_{2} \mathrm{O}, \mathbf{4}$ and $\mathbf{5}$.

Table S1. Molar magnetic susceptibilities in solution ${ }^{a}$ and in the solid state for compounds 3-D·Et $2 \mathrm{O}, \mathbf{5 -}$ D, 6-D and 7-D at $302 \mathrm{~K}$.

\begin{tabular}{|c|c|c|}
\hline Compound & $\chi_{\mathrm{m}}($ solution $)$ & $\chi_{\mathrm{m}}(\text { solid state })^{b}$ \\
\hline 3-D· $\mathrm{Et}_{2} \mathrm{O}$ & 0.0429 & 0.0422 \\
\hline 5-D & 0.0409 & 0.0404 \\
\hline 6-D & 0.0218 & 0.0214 \\
\hline 7-D & 0.0146 & $0.0145^{c}$ \\
\hline
\end{tabular}

${ }^{a}$ Determined using ${ }^{2} \mathrm{H}-\mathrm{NMR}$ Evans method, 3\% w/w $\mathrm{CDCl}_{3}$ in $\mathrm{Et}_{2} \mathrm{O}, 302 \mathrm{~K}$. ${ }^{b}$ Extrapolated from experimental datapoints recorded on the nondeuterated variant, unless otherwise stated. ${ }^{c}$ Calculated from Curie law, with $S=5 / 2$ and $g=2.0023$.

\section{Complete author list for reference 29}

(29) Abbati, G. L.; Brunel, L.-C.; Casalta, H.; Cornia, A.; Fabretti, A. C.; Gatteschi, D.; Hassan, A. K.; Jansen, A. G. M.; Maniero, A. L.; Pardi, L.; Paulsen, C.; Segre, U. Chem. Eur. J. 2001, 7, 1796. 